\title{
E-selectin gene haplotypes are associated with the risk of myocardial infarction
}

Jarosław Gorący ${ }^{1}$, Mariusz Kaczmarczyk ${ }^{2}$, Andrzej Ciechanowicz ${ }^{2}$, Krzysztof Safranow ${ }^{3}$, Joanna Gorący², Katarzyna Jakubowska ${ }^{3}$, Dariusz Chlubek ${ }^{3}$, Iwona Gorący²

\begin{abstract}
${ }^{1}$ Clinic of Cardiology, Pomeranian Medical University, Szczecin, Poland
2Department of Clinical and Molecular Biochemistry, Pomeranian Medical University, Szczecin, Poland

${ }^{3}$ Department of Biochemistry and Medical Chemistry, Pomeranian Medical University, Szczecin, Poland
\end{abstract}

Submitted: 30 August 2018

Accepted: 28 November 2018

Arch Med Sci 2019; 15 (5): 1223-1231

DOI: https://doi.org/10.5114/aoms.2019.84413

Copyright $\odot 2019$ Termedia \& Banach

\section{Abstract}

Introduction: Endothelial dysfunction is one of the most important factors implicated in the pathogenesis of coronary artery disease (CAD). The aim of this study was to investigate the association of the E-selectin gene (SELE) with CAD and CAD-related traits using tagging polymorphisms.

Material and methods: A total of 379 Polish patients who had undergone angiography were included: 261 patients with angiographically documented CAD, 202 CAD patients without myocardial infarction (CAD/MI(-) group) and 59 patients with myocardial infarction (CAD/MI(+) group) as well as 118 healthy control subjects (non-CAD). Eight tagging single nucleotide polymorphisms (SNPs) in the SELE gene were selected using genotype data from HapMap. Genotyping was performed using PCR-RFLP and PCR-DHPLC methods. Results: The most common SELE haplotype in this analysis ([C;G;T;C;G;T], $31.2 \%)$ showed a negative association with myocardial infarction (MI) (CAD/ $\mathrm{MI}(+)$ vs. non-CAD) under the additive $(p=0.001)$, dominant $(p=0.006)$ and recessive $(p=0.012)$ model. Two other haplotypes ([C;G;C;C;A;C], [C;A;C;A;G;T], 5.73\% and $18.1 \%$, respectively) were also negatively associated with $\mathrm{MI}$ under the additive and dominant model. We also found two haplotypes ([T;G;T;C;G;T], [C;G;C;C;A;T], $1.52 \%$ and $6.71 \%$, respectively) associated with the risk for $\mathrm{MI}(\mathrm{CAD} / \mathrm{MI}(+)$ vs. CAD/MI(-)), acting in both additive $(p=0.04, p=0.007$, respectively) and dominant $(p=0.04, p=0.004$, respectively) manner. There was no association with either CAD/MI(-) or with severity of CAD expressed as the number of vessels involved.

Conclusions: Our results suggest that SELE is one of the independent genetic factors modifying the risk of myocardial infarction.

Key words: coronary artery disease, E-selectin gene, haplotypes, myocardial infarction, tagging single nucleotide polymorphisms.

\section{Introduction}

Cardiovascular diseases, including coronary artery disease (CAD) and myocardial infarction (MI), remain important mortality and morbidity factors worldwide. Coronary artery disease is one with a complicated background, resulting from traditional and genetic risk factors and their interaction [1-4]. Endothelial dysfunction is the most important factor in the pathogenesis of CAD. Leukocyte adhesion to the wall of the activated

\author{
Corresponding author: \\ Iwona Gorący MD, PhD \\ Department of Clinical \\ and Molecular \\ Biochemistry \\ Pomeranian Medical \\ University \\ Al. Powstańców Wlkp. 72 \\ 70-111 Szczecin, Poland \\ Phone: +48 4661495 \\ E-mail: igor@pum.edu.pl
}


blood vessel endothelium has proved to be one of the initial features of arteriosclerosis. Proinflammatory adhesion molecules such as selectins, integrins, immunoglobulins and chemokines modulate this process [5]. Leukocytes are captured from the bloodstream and then rolled along the endothelial cell surface. E-selectin is expressed on the activated endothelium and plays the main role in monocyte migration as a rolling mediating factor. It is the first stage for endothelial monocyte and leukocyte adhesion and transmigration $[6,7]$.

The E-selectin gene (SELE) is located on chromosome 1q22-q25. Genetic polymorphisms at the SELE locus may regulate the gene expression levels and affect the biological function of the protein $[8,9]$. The SELE gene was determined with many single nucleotide polymorphisms and several polymorphisms of the E-selectin gene have been mentioned to contribute to CAD, hypertension and ischemic cerebrovascular disease [10-12]. Most studies have extensively tested two polymorphisms in particular, A561C (rs5361) and G98T (rs1805193), which have been implicated in susceptibility to CAD $[13,14]$. These functional variants seem to play an important role in modifying the secondary structure of E-selectin by exchanging amino acids and regulating cell-cell interactions $[15,16]$. Other variations of SELE may also be associated with atherosclerosis or hypertension $[17,18]$. We previously showed that the low frequency haplotypes of the E-selectin gene may reduce susceptibility to coronary artery diseases in the Polish population [19]. Although there are many reports confirming a correlation between genetic alterations of SELE and inter-individual variation in the soluble E-selectin (sE-selectin), the relationship between the two still remains controversial [20, 21].

Moreover, it has been established that SELE gene polymorphism, which predisposes to chronic inflammation development/progression, can affect the conditions with underlying inflammatory disorders [22]. According to in vivo studies, E-selectin expression can be elevated continuously as a result of chronic localized inflammation [23], whereas without coexisting inflammation, expression of E-selectin remains at negligible concentrations or it is indeterminable $[24,25]$. The pivotal role in the inflammatory process and endothelial function indicate that the SELE gene is a plausible candidate in CAD development. It must be noted, however, that variability across the E-selectin gene and its association with coronary artery disease remain poorly investigated and the previous studies have mostly concentrated on single gene variants.

Therefore, the purpose of this study was to investigate the association between SELE haplo- type tagging polymorphisms and coronary artery disease with or without a history of MI, as well as severity of CAD based on the number of coronary vessels involved in a Polish angiographically characterized population.

\section{Material and methods}

The study was performed in a cohort of Polish subjects ( $n=379$ ) recruited randomly from the inpatient Cardiology Department, Pomeranian Medical University, Szczecin, Poland, including 261 patients with angiographically documented coronary artery disease (CAD) defined as $\geq 50 \%$ stenosis of at least one major coronary artery. There were 59 patients with a history of myocardial infarction (CAD/MI(+) group), diagnosed according to recommendations of the Joint European Society of Cardiology/American College of Cardiology Committee. The CAD patients without a history of myocardial infarction (CAD/MI(-), $n=202$ ) were further divided into multi-vessel CAD (MCAD, $n=123)$ and single-vessel CAD (SCAD, $n=79)$ disease. The control group included 118 participants without CAD confirmed by coronary angiography (non-CAD group). Patients with clinical diagnosis of cardiomyopathy, coagulopathy, collagenosis and chronic inflammatory disease were excluded from the study. The protocol of the study was approved by the Ethics Committee of the Pomeranian Medical University, with formal informed consent signed by all the participants.

Demographic data were collected during the clinical trial. Hypertension, diabetes and smoking addiction were defined. Routine biochemical blood analyses were done including total cholesterol (TCH), triacylglycerol (TG), HDL cholesterol (HDL-C) and LDL cholesterol (LDL-C). Coronary angiography was performed according to standard procedures using Philips INTEGRIS HM 3000 (Philips, Netherlands) and Philips ALURA (Philips, Netherlands) devices. Significant stenosis was defined based on American Heart Association criteria as $\geq 50 \%$ stenosis of the coronary artery lumen.

The genotype data dump file for the CEU (Utah residents with northern and western European ancestry) population (chromosome 1: 166,423,439 - 166,434,836, HapMap release 21, NCBI Human Genome Build 35) was downloaded from the HapMap Genome Browser. The selection of tagging single nucleotide polymorphisms (SNPs) was conducted on HapMap data spanning a region of $11 \mathrm{~kb}$ using Haploview's Tagger in the pairwise mode, $r^{2}$ threshold of 0.8 and the minimum allele frequency of $5 \%$. Eight markers were selected out of 30 SNPs by the tagging algorithm: C1901T (rs3917454, NM_000450.2:C.529+123C>T),A2252G(rs3917412, NM_000450.2:c.529+427A>G), A2692G (rs3917417, NM_000450.2:c.530-304G>A), C2935T (rs3917419, 
NM_000450.2:c.530-61C>T), A3929C (rs1534904, NM_000450.2:c.716-11A>C), G5310A (rs1076637, NM_000450.2:c.1091-57G>A), T7836C (rs5356, NM_000450.2:c.1800T>C) and C8304T (rs3917438, NM_000450.2:c.*16-200C >T). Genomic DNA was extracted from peripheral blood leukocytes using the QIAamp DNA Mini Kit (QIAGEN, Germany). Seven tagging SNPs were genotyped using a polymerase chain reaction - restriction fragment length polymorphism (PCR-RFLP) method (Table I). The products of digestion were separated in $3 \%$ agarose gels. The rs3917417 was genotyped using PCR-DHPLC (with primers 5'-AgACAgTgCAgCATTAgggTTTTA and 5'-TTCTACCCCTTTTCTTTTATTCAA). DHPLC analysis was performed using a Hewlett-Packard 1050/1100 chromatographic system (Agilent, Waldbronn, Germany).

\section{Statistical analysis}

Quantitative data (age, serum lipids) were presented as the mean and standard deviation or median and interquartile range depending on the distribution, which was assessed using the Kolmogorov-Smirnov test. Demographic, clinical and biochemical characteristics of patients were compared using the $t$-test or $\chi^{2}$ test (Statistica, Dell Inc. (2016), version 13, software. dell.com). Agreement with Hardy-Weinberg expectations was checked using the $\chi^{2}$ test with 1 degree of freedom. The haplotype analysis was conducted using the haplo.stats package in $\mathrm{R}$ (https://cran.r-project.org). The haplo.score function was used to compute both omnibus and haplotype-specific score statistics to test the association between SELE haplotypes and binary traits allowing for adjustment for traditional risk factors. As there was a correlation between serum lipids, only triacylglycerol (logarithm) was used as a covariate (in addition to other covariates).
Only haplotypes with estimated frequency of at least $1 \%$ were considered. Three haplotype effects were examined: additive (the number of copies of a particular haplotype), dominant (homozygotes and heterozygotes were assumed to have the same effect), recessive (only homozygotes for a particular haplotype have an effect). A $p<0.05$ was considered significant.

\section{Results}

The clinical and biochemical characteristics of the CAD $(n=261)$ and non-CAD patients $(n=118)$ are summarized in Table II. Two tag SNPS, c.530304G $>$ A and C. ${ }^{*} 16-200 C>T$, deviated significantly from HWE expectations in the non-CAD group and were excluded from further analyses (Table III). The results from the association analysis between the 6-marker SELE haplotypes (NM_000450.2:C. $[529+123 \mathrm{C}>\mathrm{T} ; \quad 529+474 \mathrm{~A}>\mathrm{G} ; \quad 530-61 \mathrm{C}>\mathrm{T} ; \quad$ 716$11 \mathrm{~A}>\mathrm{C} ; 1091-57 \mathrm{G}>\mathrm{A} ;$ 1800T>C]) and the CAD-related traits are presented in Tables IV-VIII. There were no differences in the SELE haplotype frequencies in the CAD group and in the CAD/MI(-) group as compared with non-CAD group (Tables $\mathrm{VI}$ and $\mathrm{VII}$ ). Also, there was no association of the SELE haplotypes with severity of CAD in the CAD/ MI(-) group expressed as the number of vessels involved (Table VIII).

We found an association of the SELE haplotypes with a history of myocardial infarction among CAD patients (CAD/MI(+) vs. non-CAD, CAD/MI(+) vs. CAD/MI(-) (Tables IV and V). Thirty-five haplotypes were reconstructed in $59 \mathrm{CAD} / \mathrm{MI}(+)$ and 118 non-CAD patients. Of these, 6 haplotypes had frequencies greater than $5 \%$ (accounting for $80.1 \%$ ), whereas 10 inferred haplotypes had frequencies greater than 1\% (87.6\%) (Table IV). Among those 10 haplotypes with frequencies greater than $1 \%$, three haplotypes ([C;G;T;C;G;T], [C;G;C;C;A;C],

Table I. Characteristics of primers, restriction enzymes and products (restriction fragments)

\begin{tabular}{|lccc|}
\hline SNP & Primers & Restriction enzyme & Products [bp] \\
\hline rs3917454 & 5'gCA gAT ggT gTC ATA Tgg CgA T & C: 214,22 \\
& 5'CgC Agg Gac ACA gAA TTA CAg TTT A & Pvu I $: 236$ \\
\hline rs3917412 & 5'AAg ACg TTg Tag AA TgA gT & Nmu C I & A: $607,191,101,132$ \\
& 5'TTg CAg gCT ggA ATA ggA g & G: $607,607,132$ \\
\hline rs3917419 & 5'AAg ACg TTg Tag AA TgA gT & C: $548,240,124,120$ \\
& 5'TTg CAg gCT ggA ATA ggA g & T: $672,240,120$ \\
\hline rs1534904 & 5' CAA TgT ATA TTg CCA ACC CAg TA & A: $363,245,271$ \\
\hline rs1076637 & 5'ATT AgC TTg CCC ATT TCC AgT AT & C: 363,516 \\
\hline rs5356 & 5'gAA CTg ggT TCA CTC AAC AAg C & Dra I : 23,274 \\
\hline rs3917438 & 5AA TAA AAT AAA AAC AAA GAC TgT ATgT A & Hin 6 I & A: 297 \\
& 5'gCT gCC AAA gCC TTg AAT CAC A & Hin 1 II & T: $24,98,58$ \\
5'CTC CCC TgC TCC CTC CCT AAg AT & & C: 122,58 \\
\hline
\end{tabular}

Restriction enzymes (MBI Fermentas, Vilnius, Lithuania). 
Table II. Clinical and biochemical characteristics of patients with coronary artery disease and control individuals

\begin{tabular}{|c|c|c|c|c|c|c|}
\hline Parameter & $\begin{array}{c}\text { CAD } \\
(n=261)\end{array}$ & $\begin{array}{l}\text { CAD/MI(-) } \\
(n=202)\end{array}$ & $\begin{array}{l}\text { CAD/MI }(+) \\
(n=59)\end{array}$ & $\begin{array}{c}\text { MCAD } \\
(n=123)\end{array}$ & $\begin{array}{c}\text { SCAD } \\
(n=79)\end{array}$ & $\begin{array}{l}\text { Non-CAD } \\
(n=118)\end{array}$ \\
\hline Age [years] & $56.4 \pm 9.3$ & $57.2 \pm 9.0$ & $53.7 \pm 9.5$ & $58.7 \pm 9.4^{*}$ & $54.9 \pm 8.0$ & $55.3 \pm 9.5$ \\
\hline Sex, & $\begin{array}{c}44 / 217^{\star} \\
16.9 \% / 83.1 \%\end{array}$ & $\begin{array}{c}36 / 166^{*} \\
17.8 \% / 82.2 \%\end{array}$ & $\begin{array}{c}8 / 51^{*} \\
13.6 \% / 86.4 \%\end{array}$ & $\begin{array}{c}21 / 102^{*} \\
17.1 \% / 82.9 \%\end{array}$ & $\begin{array}{c}15 / 64^{*} \\
19.0 \% / 81.0 \%\end{array}$ & $\begin{array}{c}49 / 69 \\
41.5 \% / 58.5 \%\end{array}$ \\
\hline BMI $\left[\mathrm{kg} / \mathrm{m}^{2}\right]$ & $27.5 \pm 3.7$ & $27.7 \pm 3.7^{\star}$ & $26.6 \pm 3.6$ & $27.7 \pm 3.6^{*}$ & $27.8 \pm 3.7$ & $26.7 \pm 4.2$ \\
\hline $\begin{array}{l}\text { Smoking, } \\
\text { Yes/no }\end{array}$ & $\begin{array}{c}111 / 150^{*} \\
42.5 \% / 57.5 \% \\
\end{array}$ & $\begin{array}{c}75 / 127^{*} \\
37.1 \% / 62.9 \% \\
\end{array}$ & $\begin{array}{c}36 / 23^{*} \\
61.0 \% / 39.0 \% \\
\end{array}$ & $\begin{array}{c}46 / 77^{\star} \\
37.4 \% / 62.6 \% \\
\end{array}$ & $\begin{array}{c}29 / 50^{*} \\
36.7 \% / 63.3 \% \\
\end{array}$ & $\begin{array}{c}27 / 91 \\
22.9 \% / 77.1 \% \\
\end{array}$ \\
\hline $\begin{array}{l}\text { Diabetes mellitus, } \\
\text { Yes/no }\end{array}$ & $\begin{array}{c}43 / 218 \\
16.5 \% / 83.5 \% \\
\end{array}$ & $\begin{array}{c}37 / 165^{*} \\
18.3 \% / 81.7 \% \\
\end{array}$ & $\begin{array}{c}6 / 53 \\
10.2 \% / 89.8 \% \\
\end{array}$ & $\begin{array}{c}28 / 95^{*} \\
22.8 \% / 77.2 \% \\
\end{array}$ & $\begin{array}{c}9 / 70^{*} \\
11.4 \% / 88.6 \% \\
\end{array}$ & $\begin{array}{c}11 / 107 \\
9.3 \% / 90.7 \% \\
\end{array}$ \\
\hline $\begin{array}{l}\text { Hypertension, } \\
\text { Yes/No }\end{array}$ & $\begin{array}{c}152 / 109 \\
58.2 \% / 41.8 \% \\
\end{array}$ & $\begin{array}{c}124 / 78^{*} \\
61.4 \% / 38.6 \% \\
\end{array}$ & $\begin{array}{c}28 / 31 \\
47.5 \% / 52.5 \% \\
\end{array}$ & $\begin{array}{c}81 / 42 \\
65.9 \% / 34.1 \% \\
\end{array}$ & $\begin{array}{c}43 / 36^{\star} \\
54.4 \% / 45.6 \% \\
\end{array}$ & $\begin{array}{c}59 / 59 \\
50.0 \% / 50 \% \\
\end{array}$ \\
\hline Triac) & $\begin{array}{c}174 \\
(132-215)^{*}\end{array}$ & $\begin{array}{c}169 \\
(123-216)^{*}\end{array}$ & $\begin{array}{c}180 \\
(156-210)^{*}\end{array}$ & $\begin{array}{c}174 \\
(120-217)^{*}\end{array}$ & $\begin{array}{c}165 \\
(126-209)^{*} \\
\end{array}$ & $\begin{array}{c}147.0 \\
(103-197) \\
\end{array}$ \\
\hline Total cholesterol [mg/dl] & $225 \pm 40$ & $225 \pm 42$ & $226 \pm 33$ & $227 \pm 43$ & $222 \pm 41$ & $220 \pm 37$ \\
\hline Cholesterol LDL [mg/dl] & $138 \pm 32$ & $138 \pm 34$ & $136 \pm 25$ & $140 \pm 36^{*}$ & $135 \pm 31$ & $132 \pm 29$ \\
\hline Cholesterol HDL [mg/dl] & $\begin{array}{c}42.0 \\
(37.0-47.0)^{\star}\end{array}$ & $\begin{array}{c}42.0 \\
(37.0-47.0)^{\star}\end{array}$ & $\begin{array}{c}53.0 \\
(47.0-62.0)^{\star}\end{array}$ & $\begin{array}{c}42.0 \\
(37.0-46.0)^{\star}\end{array}$ & $\begin{array}{c}43.0 \\
(38.0-49.0)^{\star}\end{array}$ & $\begin{array}{c}47.0 \\
(39.0-56.0)\end{array}$ \\
\hline
\end{tabular}

*Significant difference as compared with non-CAD patients, CAD - coronary artery disease, CAD/MI(+) - patients with a history of myocardial infarction, CAD/MI(-) - patients without a history of myocardial infarction, MCAD - multi-vessel coronary artery disease, $S C A D$ - single-vessel coronary artery disease, non-CAD - absence of coronary artery disease; mean \pm standard deviations or median with quartiles (in brackets). Arterial hypertension was defined as: systolic blood pressure exceeding $140 \mathrm{~mm} \mathrm{Hg}$, or diastolic blood pressure greater than $90 \mathrm{~mm} \mathrm{Hg}$; Diagnosed diabetes or fasting glucose $\geq 6.9 \mathrm{mmol} / \mathrm{l}$; Smoking - patients were classified as "current smokers" if they reported a daily rate of more than five cigarettes, otherwise patients were classified as "non-smokers."

[C;A;C;A;G;T]) were associated (based on adjusted $p$-values) with a history of myocardial infarction under additive and dominant models and one haplotype [C;G;T;C;G;T] under a recessive model (Table IV). The most common SELE haplotype in this analysis ([C;G;T;C;G;T], 31.2\%) was negatively associated with myocardial infarction under the assumption of additive, dominant and recessive effects. Its frequency in the patients with a history of myocardial infarction was 3.0 times lower than in non-CAD individuals (12.7\% vs. $37.5 \%)$. Two other haplotypes ([C;G;C;C;A;C], [C;A;C;A;G;T]) showed a negative association effect under the assumption of additive and dominant models. The haplotype $[C ; G ; C ; C ; A ; C]$ was detected only in the non-CAD patients with a frequency of $8.81 \%$.

Table III. Single nucleotide polymorphisms of the SELE gene included in the study

\begin{tabular}{|lcccccc|}
\hline SNP (reference sequence & $\begin{array}{c}\text { CAD } \\
\text { NM_000450.2) }\end{array}$ & $\begin{array}{c}\text { CAD/MI(-) } \\
(n=261)\end{array}$ & $\begin{array}{c}\text { CAD/MI(+) } \\
(n=59)\end{array}$ & $\begin{array}{c}\text { MCAD } \\
(n=123)\end{array}$ & $\begin{array}{c}\text { SCAD } \\
(n=79)\end{array}$ & $\begin{array}{c}\text { Non-CAD } \\
(n=118)\end{array}$ \\
\hline c.529+123C>T, & $219 / 40 / 2$ & $180 / 22 / 0$ & $39 / 18 / 2$ & $111 / 12 / 0$ & $69 / 10 / 0$ & $113 / 5 / 0$ \\
CC/CT/TT & $\chi^{2}=0.01$ & $\chi^{2}=0.67$ & $\chi^{2}=0$ & $\chi^{2}=0.32$ & $\chi^{2}=0.36$ & $\chi^{2}=0.06$ \\
\hline c.529+474A $>$ G, & $16 / 106 / 139$ & $11 / 87 / 104$ & $5 / 19 / 35$ & $7 / 50 / 66$ & $4 / 37 / 38$ & $9 / 40 / 69$ \\
AA/AG/GG & $\chi^{2}=0.51$ & $\chi^{2}=1.75$ & $\chi^{2}=1.02$ & $\chi^{2}=0.39$ & $\chi^{2}=1.77$ & $\chi^{2}=0.87$ \\
\hline c.530-304G>A, & $210 / 51 / 0$ & $162 / 40 / 0$ & $48 / 11 / 0$ & $99 / 24 / 0$ & $63 / 16 / 0$ & $95 / 19 / 4$ \\
GG/GA/AA & $\chi^{2}=3.06$ & $\chi^{2}=2.44$ & $\chi^{2}=0.62$ & $\chi^{2}=1.44$ & $\chi^{2}=1$ & $\chi^{2}=4.98^{\ddagger}$ \\
\hline c.530-61C>T, & $89 / 136 / 36$ & $65 / 109 / 28$ & $24 / 27 / 8$ & $40 / 66 / 17$ & $25 / 43 / 11$ & $43 / 51 / 24$ \\
CC/CT/TT & $\chi^{2}=1.97$ & $\chi^{2}=2.75$ & $\chi^{2}=0.01$ & $\chi^{2}=1.54$ & $\chi^{2}=1.21$ & $\chi^{2}=1.5$ \\
\hline c.716-11A>C, & $30 / 131 / 100$ & $23 / 100 / 79$ & $7 / 31 / 21$ & $16 / 56 / 51$ & $7 / 44 / 28$ & $13 / 45 / 60$ \\
AA/AC/CC & $\chi^{2}=1.74$ & $\chi^{2}=1.06$ & $\chi^{2}=0.76$ & $\chi^{2}=0.01$ & $\chi^{2}=3.12$ & $\chi^{2}=1.03$ \\
\hline c. 1091-57G>A, & $195 / 58 / 8$ & $156 / 44 / 2$ & $37 / 16 / 6$ & $95 / 27 / 1$ & $61 / 17 / 1$ & $82 / 31 / 5$ \\
GG/GA/AA & $\chi^{2}=1.96$ & $\chi^{2}=0.33$ & $\chi^{2}=3.71$ & $\chi^{2}=0.38$ & $\chi^{2}=0.02$ & $\chi^{2}=0.85$ \\
\hline c.1800T>C, & $211 / 46 / 4$ & $168 / 32 / 2$ & $43 / 14 / 2$ & $102 / 21 / 0$ & $66 / 11 / 2$ & $95 / 21 / 2$ \\
TT/TC/CC & $\chi^{2}=0.65$ & $\chi^{2}=0.12$ & $\chi^{2}=0.4$ & $\chi^{2}=1.07$ & $\chi^{2}=2.84$ & $\chi^{2}=0.43$ \\
\hline c. ${ }^{*} 16-200 C>T$, & $240 / 21 / 0$ & $186 / 16 / 0$ & $54 / 5 / 0$ & $117 / 6 / 0$ & $69 / 10 / 0$ & $107 / 9 / 2$ \\
CC/CT/TT & $\chi^{2}=0.46$ & $\chi^{2}=0.34$ & $\chi^{2}=0.12$ & $\chi^{2}=0.08$ & $\chi^{2}=0.36$ & $\chi^{2}=8.43^{\dagger}$ \\
\hline
\end{tabular}

Significant deviation from HWE expectations as tested using $\chi^{2}$ with 1 degree of freedom: ${ }^{\dagger} p=0.004,{ }^{\ddagger} p=0.03$, CAD - coronary artery disease, CAD/MI(+) - patients with a history of myocardial infarction, CAD/MI(-) - patients without a history of myocardial infarction, $M C A D$ - multi-vessel coronary artery disease, $S C A D$ - single-vessel coronary artery disease, non-CAD - absence of coronary artery disease. 
Table IV. SELE haplotype (NM 000450.2:C.[529+123C>T; 529+474A>G; 530-61C>T; 716-11A>C; 1091-57G>A; $1800 T>C])$ association analysis in patients with a history of CAD/MI $(+)(n=59)$ and non-CAD control individuals $(n=118)$

\begin{tabular}{|c|c|c|c|c|c|c|c|c|c|}
\hline \multirow[t]{2}{*}{ Haplotype } & \multicolumn{3}{|c|}{ Frequency (\%) } & \multicolumn{2}{|c|}{ Additive } & \multicolumn{2}{|c|}{ Dominant } & \multicolumn{2}{|c|}{ Recessive } \\
\hline & Total $^{\ddagger}$ & $\begin{array}{l}\text { CAD/ } \\
\text { MI(+) }\end{array}$ & $\begin{array}{l}\text { Non- } \\
\text { CAD }\end{array}$ & Score & $\begin{array}{c}P^{\dagger} \\
<0.001^{*}\end{array}$ & Score & $\begin{array}{c}P^{\dagger} \\
<0.001^{*}\end{array}$ & Score & $\begin{array}{c}P^{\dagger} \\
0.007^{\star}\end{array}$ \\
\hline$[\mathrm{C} ; \mathrm{G} ; \mathrm{T} ; \mathrm{C} ; \mathrm{G} ; \mathrm{T}]$ & 31.2 & 12.7 & 37.5 & -3.46 & 0.001 & -2.82 & 0.006 & -3.06 & 0.012 \\
\hline$[C ; G ; C ; C ; A ; C]$ & 5.73 & 0 & 8.81 & -3.18 & 0.0007 & -3.18 & 0.0007 & - & - \\
\hline$[C ; A ; C ; A ; G ; T]$ & 18.1 & 6.72 & 23.0 & -2.98 & 0.01 & -2.73 & 0.02 & -2.05 & 0.10 \\
\hline [C;G;T;C;A;T] & 1.81 & 0 & 2.25 & -0.63 & 0.48 & -0.47 & 0.32 & - & - \\
\hline$[C ; G ; C ; C ; G ; T]$ & 10.3 & 9.31 & 10.7 & -0.49 & 0.92 & -0.56 & 0.74 & - & - \\
\hline$[\mathrm{C} ; \mathrm{G} ; \mathrm{T} ; \mathrm{C} ; \mathrm{G} ; \mathrm{C}]$ & 1.38 & 2.54 & 1.24 & 0.53 & 0.37 & NA & - & - & - \\
\hline$[\mathrm{T} ; \mathrm{G} ; \mathrm{C} ; \mathrm{C} ; \mathrm{G} ; \mathrm{T}]$ & 2.62 & 4.58 & 2.12 & 1.30 & 0.38 & 1.30 & 0.38 & - & - \\
\hline$[C ; G ; C ; C ; A ; T]$ & 7.65 & 11.6 & 6.32 & 1.39 & 0.14 & 1.39 & 0.14 & - & - \\
\hline$[C ; G ; C ; A ; G ; T]$ & 7.12 & 12.9 & 5.65 & 1.66 & 0.17 & 1.44 & 0.27 & - & - \\
\hline$[C ; A ; C ; C ; G ; T]$ & 1.74 & 3.48 & 0.90 & 2.33 & 0.13 & 2.33 & 0.13 & - & - \\
\hline
\end{tabular}

${ }^{\dagger} P$-value adjusted for sex, age, BMI, smoking, diabetes mellitus, hypertension, triacylglycerol (logarithm), *omnibus haplotype test statistic p-value, NA - not applicable. ${ }^{\ddagger} T o t a l-C A D / M I(+)$ group and non-CAD group, CAD/MI(+) - patients with a history of myocardial infarction, non-CAD - absence of coronary artery disease.

Thirty-seven SELE haplotypes were reconstructed in 59 CAD/MI(+) and 202 CAD/MI(-) patients. Of these, 5 haplotypes with frequencies greater than $5 \%$ accounted for $77.4 \%$ of all haplotypes, whereas 12 haplotypes with frequencies above $1 \%$ accounted for $92.9 \%$ (Table V). Similarly to a previous anal- ysis, we identified three negatively associated haplotypes [C;G;T;C;G;T], [C;A;C;A;G;T], [C;G;C;C;A;C] that overlapped with haplotypes inferred for CAD/MI(+) and non-CAD patients (Table IV). The most common negatively associated haplotype $[\mathrm{C} ; \mathrm{G} ; \mathrm{T} ; \mathrm{C} ; \mathrm{G} ; \mathrm{T}]$ was 2.9 times less frequent in

Table V. SELE haplotype (NM_000450.2:c.[529+123C>T; 529+474A>G; 530-61C>T; 716-11A>C; 1091-57G>A; $1800 \mathrm{~T}>\mathrm{C}])$ association analysis in CAD/MI $(+)(n=59)$ and CAD/MI $(-)(n=202)$

\begin{tabular}{|c|c|c|c|c|c|c|c|c|c|}
\hline \multirow[t]{2}{*}{ Haplotype } & \multicolumn{3}{|c|}{ Frequency (\%) } & \multicolumn{2}{|c|}{ Additive } & \multicolumn{2}{|c|}{ Dominant } & \multicolumn{2}{|c|}{ Recessive } \\
\hline & Total‡ & $\begin{array}{l}\text { CAD/ } \\
\text { MI(+) }\end{array}$ & $\begin{array}{l}\text { CAD/ } \\
\text { MI(-) }\end{array}$ & Score & $\begin{array}{c}P^{\dagger} \\
<0.001^{*}\end{array}$ & Score & $\begin{array}{c}P^{\dagger} \\
<0.001^{*}\end{array}$ & Score & $\begin{array}{c}P^{\dagger} \\
0.02^{\star}\end{array}$ \\
\hline [C;G;T;C;G;T] & 31.8 & 12.7 & 36.3 & -4.05 & $<0.001$ & -4.02 & $<0.001$ & -2.19 & 0.02 \\
\hline$[C ; A ; C ; A ; G ; T]$ & 20.3 & 6.72 & 24.1 & -3.60 & 0.001 & -3.53 & 0.002 & -1.65 & 0.11 \\
\hline$[C ; G ; C ; C ; A ; C]$ & 4.81 & 0 & 6.41 & -2.75 & 0.006 & -2.75 & 0.006 & NA & - \\
\hline [T;G;C;C;G;T] & 3.81 & 4.58 & 4.04 & -0.14 & 0.93 & -0.14 & 0.93 & NA & - \\
\hline$[C ; G ; C ; A ; G ; T]$ & 10.4 & 12.9 & 10.1 & 0.32 & 0.59 & 0.32 & 0.68 & NA & - \\
\hline [C;A;T;A;G;T] & 1.46 & 0.81 & 1.49 & 0.39 & 0.75 & 0.77 & 0.67 & NA & - \\
\hline$[C ; G ; C ; C ; G ; T]$ & 8.16 & 9.30 & 7.48 & 0.67 & 0.68 & 0.62 & 0.71 & NA & - \\
\hline$[\mathrm{C} ; \mathrm{G} ; \mathrm{T} ; \mathrm{C} ; \mathrm{G} ; \mathrm{C}]$ & 1.21 & 2.54 & 0.88 & 0.87 & 0.32 & 0.87 & 0.32 & NA & - \\
\hline [C;A;T;C;G;T] & 1.39 & 4.46 & 1.06 & 1.01 & 0.84 & 1.01 & 0.84 & NA & - \\
\hline$[C ; G ; C ; C ; G ; C]$ & 1.33 & 2.01 & 0.96 & 1.14 & 0.44 & 1.14 & 0.44 & NA & - \\
\hline$[\mathrm{T} ; \mathrm{G} ; \mathrm{T} ; \mathrm{C} ; \mathrm{G} ; \mathrm{T}]$ & 1.52 & 5.24 & 1.16 & 2.42 & 0.04 & 2.42 & 0.04 & NA & - \\
\hline$[C ; G ; C ; C ; A ; T]$ & 6.71 & 11.6 & 5.19 & 2.60 & 0.007 & 2.86 & 0.004 & NA & - \\
\hline
\end{tabular}

${ }^{\dagger} P$-value adjusted for sex, age, BMI, smoking, diabetes mellitus, hypertension, triacylglycerol (logarithm), *omnibus haplotype test statistic p-value, NA - not applicable. ${ }^{\ddagger} T o t a l-C A D / M I(+)$ group and CAD/MI(-) group, CAD/MI(+) - patients with a history of myocardial infarction, CAD/MI(-) - patients without a history of myocardial infarction. 
the $\mathrm{CAD} / \mathrm{MI}(+)$ group compared with $\mathrm{CAD} / \mathrm{MI}(-)$ patients (12.7\% vs. 36.3\%), while [C;G;C;C;A;C] occurred only in $\mathrm{CAD} / \mathrm{MI}(-)$ patients ( $0 \%$ vs. $6.41 \%)$. In addition, we found two haplotypes associated with a history of myocardial infarction ([T;G;T;C;G;T], [C;G;C;C;A;T]) acting in both additive and dominant manner (Table $\mathrm{V})$.

\section{Discussion}

In the current study we conducted a haplotype-based analysis of the SELE gene in 261 patients with coronary artery disease (including 59 with myocardial infarction) and 118 control in- dividuals with no significant coronary stenosis. To the best of our knowledge this is the first study investigating the association of the SELE gene with the risk of coronary artery disease and related phenotypes that uses a tagging SNPs approach for the selection of genetic variants.

Our main findings are as follows:

- We identified two SELE haplotypes ([T;G;T;C;G;T] and $[C ; G ; C ; C ; A ; T]$ ) that were significantly more common in patients with a history of myocardial infarction than in non-MI CAD patients, thereby possibly increasing the risk of $\mathrm{MI}$ among patients with coronary artery disease.

Table VI. SELE haplotype (NM 000450.2:C.[529+123C>T; 529+474A>G; 530-61C>T; 716-11A>C; 1091-57G>A; $1800 T>C])$ association analysis in patients with CAD $(n=261)$ and non-CAD control individuals $(n=118)$

\begin{tabular}{|c|c|c|c|c|c|c|c|c|c|}
\hline \multirow[t]{2}{*}{ Haplotype } & \multicolumn{3}{|c|}{ Frequency (\%) } & \multicolumn{2}{|c|}{ Additive } & \multicolumn{2}{|c|}{ Dominant } & \multicolumn{2}{|c|}{ Recessive } \\
\hline & Total $^{\ddagger}$ & CAD & $\begin{array}{l}\text { Non- } \\
\text { CAD }\end{array}$ & Score & $\begin{array}{c}P^{\dagger} \\
0.02^{*}\end{array}$ & Score & $\begin{array}{c}P^{\dagger} \\
0.17^{\star}\end{array}$ & Score & $\begin{array}{c}P^{\dagger} \\
0.22^{*}\end{array}$ \\
\hline$[C ; G ; C ; C ; A ; C]$ & 6.13 & 4.73 & 8.81 & -1.85 & 0.07 & -1.85 & 0.07 & NA & - \\
\hline$[\mathrm{C} ; \mathrm{G} ; \mathrm{T} ; \mathrm{C} ; \mathrm{G} ; \mathrm{T}]$ & 33.8 & 31.7 & 37.5 & -1.52 & 0.09 & -0.40 & 0.33 & -2.53 & 0.05 \\
\hline$[C ; G ; C ; C ; G ; T]$ & 9.01 & 8.14 & 10.7 & -1.08 & 0.22 & -1.09 & 0.19 & -0.43 & 0.80 \\
\hline [C;A;C;A;G;T] & 21.3 & 20.5 & 23.0 & -0.56 & 0.91 & -0.04 & 0.75 & -1.45 & 0.69 \\
\hline$[C ; G ; C ; C ; A ; T]$ & 6.17 & 6.73 & 6.32 & -0.27 & 0.93 & -0.40 & 0.71 & NA & - \\
\hline$[\mathrm{C} ; \mathrm{G} ; \mathrm{T} ; \mathrm{C} ; \mathrm{G} ; \mathrm{C}]$ & 1.29 & 1.43 & 1.24 & 0.38 & 0.67 & 0.38 & 0.67 & NA & - \\
\hline$[C ; A ; T ; A ; G ; T]$ & 1.19 & 1.41 & 0.66 & 0.54 & 0.82 & 0.39 & 0.96 & NA & - \\
\hline$[C ; G ; C ; C ; G ; C]$ & 1.06 & 1.34 & 0.12 & 1.07 & 0.10 & 1.07 & 0.10 & NA & - \\
\hline [T;G;C;C;G;T] & 3.20 & 3.78 & 2.12 & 1.31 & 0.15 & 1.31 & 0.15 & NA & - \\
\hline$[C ; G ; C ; A ; G ; T]$ & 8.80 & 10.39 & 5.65 & 1.92 & 0.14 & 1.74 & 0.19 & NA & - \\
\hline
\end{tabular}

${ }^{\dagger} P$-value adjusted for sex, age, BMI, smoking, diabetes mellitus, hypertension, triacylglycerol (logarithm), *omnibus haplotype test statistic $p$-value, NA - not applicable. ${ }^{\ddagger}$ Total - CAD group and non-CAD group, CAD - coronary artery disease, non-CAD - absence of coronary artery disease.

Table VII. SELE haplotype (NM_000450.2:C.[529+123C>T; 529+474A>G; 530-61C>T; 716-11A>C; 1091-57G>A; $1800 \mathrm{~T}>\mathrm{C}])$ association analysis in CAD/MI $(-)(n=202)$ and non-CAD control individuals $(n=118)$

\begin{tabular}{|c|c|c|c|c|c|c|c|c|c|}
\hline \multirow[t]{2}{*}{ Haplotype } & \multicolumn{3}{|c|}{ Frequency (\%) } & \multicolumn{2}{|c|}{ Additive } & \multicolumn{2}{|c|}{ Dominant } & \multicolumn{2}{|c|}{ Recessive } \\
\hline & Total $^{\ddagger}$ & $\begin{array}{l}\text { CAD/ } \\
\text { MI(-) }\end{array}$ & $\begin{array}{l}\text { Non- } \\
\text { CAD }\end{array}$ & Score & $\begin{array}{c}P^{\dagger} \\
0.35^{\star}\end{array}$ & Score & $\begin{array}{c}P^{\dagger} \\
0.41^{*}\end{array}$ & Score & $\begin{array}{c}P^{\dagger} \\
0.42^{*}\end{array}$ \\
\hline$[\mathrm{C} ; \mathrm{G} ; \mathrm{C} ; \mathrm{C} ; \mathrm{G} ; \mathrm{T}]$ & 8.96 & 7.48 & 10.7 & -1.52 & 0.10 & -1.53 & 0.09 & NA & - \\
\hline$[C ; G ; C ; C ; A ; C]$ & 7.05 & 6.41 & 8.81 & -1.00 & 0.29 & -1.00 & 0.29 & NA & - \\
\hline$[C ; G ; C ; C ; A ; T]$ & 5.39 & 5.19 & 6.32 & -0.63 & 0.64 & -0.80 & 0.42 & NA & - \\
\hline$[\mathrm{C} ; \mathrm{G} ; \mathrm{T} ; \mathrm{C} ; \mathrm{G} ; \mathrm{T}]$ & 36.9 & 36.3 & 37.5 & -0.32 & 0.54 & 0.80 & 0.93 & -1.75 & 0.19 \\
\hline [C;A;T;A;G;T] & 1.36 & 1.49 & 0.66 & 0.45 & 0.99 & 0.24 & 0.85 & NA & - \\
\hline$[\mathrm{C} ; \mathrm{A} ; \mathrm{C} ; \mathrm{A} ; \mathrm{G} ; \mathrm{T}]$ & 23.6 & 24.1 & 23.0 & 0.46 & 0.35 & 0.97 & 0.28 & -0.89 & 0.90 \\
\hline$[\mathrm{T} ; \mathrm{G} ; \mathrm{C} ; \mathrm{C} ; \mathrm{G} ; \mathrm{T}]$ & 3.31 & 4.04 & 2.12 & 1.36 & 0.10 & 1.36 & 0.10 & NA & - \\
\hline$[C ; G ; C ; A ; G ; T]$ & 8.49 & 10.1 & 5.65 & 1.71 & 0.25 & 1.51 & 0.32 & NA & - \\
\hline
\end{tabular}

${ }^{\dagger} P$-value adjusted for sex, age, BMI, smoking, diabetes mellitus, hypertension, triacylglycerol (logarithm), *omnibus haplotype test statistic p-value, NA - not applicable. ${ }^{\ddagger}$ Total - CAD/MI(-) group and non-CAD group, CAD/MI(-) - patients without a history of myocardial infarction, non-CAD - absence of coronary artery disease. 
Table VIII. SELE haplotype (NM 000450.2:C.[529+123C>T; 529+474A>G; 530-61C>T; 716-11A>C; 1091-57G>A; $1800 \mathrm{~T}>\mathrm{C}]$ ) analysis in CAD/MI $(-)$ patients with multi-vessel disease (MCAD, $n=123$ ) and single-vessel disease $(\mathrm{SCAD}, n=79)$

\begin{tabular}{|c|c|c|c|c|c|c|c|c|c|}
\hline \multirow[t]{2}{*}{ Haplotype } & \multicolumn{3}{|c|}{ Frequency (\%) } & \multicolumn{2}{|c|}{ Additive } & \multicolumn{2}{|c|}{ Dominant } & \multicolumn{2}{|c|}{ Recessive } \\
\hline & Total $^{\ddagger}$ & MCAD & SCAD & Score & $\begin{array}{c}P^{\dagger} \\
0.99^{\star}\end{array}$ & Score & $\begin{array}{c}P^{\dagger} \\
0.98^{\star}\end{array}$ & Score & $\begin{array}{c}P^{\dagger} \\
0.71^{*}\end{array}$ \\
\hline$[C ; A ; C ; A ; G ; T]$ & 24.1 & 22.0 & 25.8 & -0.57 & 0.70 & -0.83 & 0.41 & 0.36 & 0.41 \\
\hline$[\mathrm{T} ; \mathrm{G} ; \mathrm{C} ; \mathrm{C} ; \mathrm{G} ; \mathrm{T}]$ & 4.04 & 3.74 & 4.28 & -0.46 & 0.76 & -0.46 & 0.76 & NA & - \\
\hline$[C ; G ; C ; C ; A ; C]$ & 6.41 & 5.69 & 6.38 & -0.32 & 0.78 & -0.32 & 0.78 & NA & - \\
\hline [T;G;T;C;G;T] & 1.16 & 0 & 0.84 & -0.28 & 0.95 & NA & - & NA & - \\
\hline [C;G;T;C;G;T] & 36.3 & 38.0 & 38.2 & -0.003 & 0.87 & 0.12 & 0.89 & -0.18 & 0.90 \\
\hline$[C ; A ; T ; A ; G ; T]$ & 1.49 & 1.55 & 0.85 & 0.02 & 0.85 & -0.37 & 0.63 & NA & - \\
\hline$[C ; G ; C ; C ; A ; T]$ & 5.19 & 5.41 & 4.48 & 0.26 & 0.94 & 0.58 & 0.76 & NA & - \\
\hline$[\mathrm{C} ; \mathrm{A} ; \mathrm{T} ; \mathrm{C} ; \mathrm{G} ; \mathrm{T}]$ & 1.06 & 1.10 & 0 & 0.47 & 0.79 & NA & - & NA & - \\
\hline$[\mathrm{C} ; \mathrm{G} ; \mathrm{C} ; \mathrm{A} ; \mathrm{G} ; \mathrm{T}]$ & 10.1 & 10.6 & 8.41 & 0.54 & 0.71 & 0.54 & 0.66 & NA & - \\
\hline$[\mathrm{C} ; \mathrm{G} ; \mathrm{C} ; \mathrm{C} ; \mathrm{G} ; \mathrm{T}]$ & 7.48 & 8.32 & 5.89 & 0.91 & 0.20 & 0.68 & 0.29 & NA & - \\
\hline
\end{tabular}

${ }^{\dagger} P$-value adjusted for sex, age, BMI, smoking, diabetes mellitus, hypertension, triacylglycerol (logarithm), *omnibus haplotype test statistic p-value, NA - not applicable. ${ }^{\ddagger}$ Total - MCAD group and SCAD group, MCAD - multi-vessel coronary artery disease, SCAD - single-vessel coronary artery disease.

- We identified three negatively associated (possibly protective) SELE haplotypes against $\mathrm{MI}$ ([C;G;T;C;G;T], [C;A;C;A;G;T] and $[\mathrm{C} ; \mathrm{G} ; \mathrm{C} ; \mathrm{C} ; \mathrm{A} ; \mathrm{C}])$ with significantly lower frequency in patients with a history of MI than in non-CAD or no-MI CAD patients. It is worth mentioning that those risk-decreasing or risk-increasing haplotype effects for MI were independent of traditional modifiable and non-modifiable risk factors.

So far, mainly two presumably functional SELE polymorphisms, A561C (rs5361) and G98T (rs1805193), have been investigated in different ethnic groups with respect to CAD $[13,14,26]$. However, the haplotype-based analysis may offer greater power in association studies than the analysis of one SNP at a time, especially when none of the investigated SNPs is a causative marker [27]. Considering the lack of association with increased risk of CAD among Caucasians for G98T in a recent meta-analysis [13], our decision to use tag SNPs in an attempt to capture a high degree of the known common variability, instead of the standard approach to testing individual candidate SNPs, seems well justified.

Our results suggest that E-selectin gene variability may, independently of conventional risk factors, modify the risk of myocardial infarction. However, there was no clear evidence for an association in patients with CAD in whom MI did not occur. Increased levels of E-selectin were reported in both coronary artery disease [28] and myocardial infarction [29]. Atalar et al. [30] found increased levels of selectins, including E-selectin, in patients with unstable angina compared with those with stable angina or without angiographically visible occlusions. Interestingly, sE-selectin levels were higher in patients with acute myocardial infarction preceded by unstable angina compared with sudden onset of infarction [31]. Since at least part of the sE-selectin level in recent years [32], despite variation, can be attributed to SELE gene polymorphism (a borderline association of the 7-marker haplotype with soluble E-selectin [33]), it seems reasonable to propose that the risk-decreasing and risk-increasing SELE haplotype effects for MI possibly reflect extreme areas of the soluble E-selectin level continuum.

Nonetheless, it is unclear why there was no association between SELE haplotypes and the risk of coronary artery disease. None of the SELE haplotypes, except for [C;G;T;C;G;T] under the recessive model (Table $\mathrm{VI}$ ), were associated with CAD with or without a history of MI (the association of the [C;G;T;C;G;T] haplotype is likely generated by $\mathrm{MI}$ patients as it disappears in the no-MI CAD patients, Table VII). It must be noted that traditional risk factors of atherosclerosis are deeply rooted in the Polish population although it should be emphasized that there has been a slight improvement which may lessen the impact of genetic factors. Indeed, the frequency of classic risk factors such as arterial hypertension, smoking, diabetes and BMI differed significantly between CAD and control patients (Table II). The other possibility is the presence of an interaction with other systems that may modify the impact of SELE gene haplotypes on CAD susceptibility. These mechanisms, however, have not been 
fully explained yet. For example, Wu et al. [33] reported that genotypes/haplotypes of the SELE gene in Taiwanese individuals are independently associated with E-selectin and matrix metalloproteinase 9 (MMP9) levels. Metalloproteinase 9 plays an important role in the destabilization of atherosclerotic plaque [34], but a direct mechanism linking E-selectin and the MMP9 gene is not clear, and further studies are needed to clarify this relationship. It is also possible that the lack of association with susceptibility to CAD may be related to ethnic differences in the social, environmental and genetic conditions contributing towards the development of CAD [35].

However, our study has several limitations. It is important to emphasize that this is the first report demonstrating that the haplotypes capturing the majority of the E-selectin gene variation are correlated with occurrence of $\mathrm{MI}$, but there is still a lack of studies showing this association in other countries and nations. Additionally it is a relatively small sample size, so the observation need to be followed up with a much larger sample size to make a causal inference. The strength of our study lies in the clinically well characterized and homogeneous cohort of patients who had undergone coronary angiography. Homogeneity is a crucial aspect in genetic association studies as population stratification may lead to spurious findings. The geographic area from which the subjects came coincides with the West Pomerania province in Poland and its genetic structure appears to be extremely homogeneous [36]. However, we did not measure the level of E-selectin, and this is a major limitation of our study. Thus, it is not clear whether the $\mathrm{MI}$ risk-modifying effect of the SELE haplotypes is mediated through an effect on the serum E-selectin level.

In conclusion, using a tagging SNPs approach we identified both negatively associated and myocardial infarction risk-enhancing common SELE haplotypes in a homogeneous Polish angiographic cohort. However, there was no evidence of an association between the SELE gene and susceptibility to coronary artery disease without a history of MI. Our results suggest that SELE is one of the independent genetic factors modulating the risk of developing myocardial infarction.

\section{Acknowledgments}

The study was financed by the internal funding of Pomeranian Medical University, Szczecin, Poland.

\section{Conflict of interest}

The authors declare no conflict of interest.

\section{References}

1. Ross R. Atherosclerosis-an inflammatory disease. N Engl J Med 1999; 340: 115-26.

2. Franchini M, Peyvandi F, Mannuci PM. The genetic basis of coronary artery disease: from candidate genes to whole genome analysis. Trends Cardiovasc Med 2008; 18: 157-62.

3. Barbarash O, Gruzdeva O, Uchasova E, Belik E, Dyleva Y, Karetnikova V. Biochemical markers of type 2 diabetes as a late complication of myocardial infarction: a case-control study. Arch Med Sci 2017; 13: 311-20.

4. Xu LB, Zhou YF, Yao JL, et al. Apolipoprotein A1 polymorphisms and risk of coronary artery disease: a metaanalysis. Arch Med Sci 2017; 13: 813-9.

5. Blankenberg S, Barbaux S, Tiret L. Adhesion molecules and atherosclerosis. Atherosclerosis 2003; 170: 191-203.

6. Yoshida M, Takano Y, Sasaoka T, et al. E-selectin polymorphism associated with myocardial infarction causes enhanced leukocyte-endothelial interactions under flow conditions. Arterioscler Thromb Vasc Biol 2003; 23: 783-8.

7. Pigott R, Dillon LP, Hemingway IH, Gearing AJ. Soluble forms of E-selectin, ICAM-1 and VCAM-1 are present in the supernatants of cytokine activated cultured endothelial cells. Biochem Biophys Res Commun 1992; 187: 584-9.

8. Grenon SM, Aguado-Zuniga J, Hatton JP, et al. Effects of fatty acids on endothelial cells: inflammation and monocyte adhesion. J Surg Res 2012; 177: e35-43.

9. Wenzel K, Felix S, Kleber FX, et al. E-selectin polymorphism and atherosclerosis: an association study. Hum Mol Genet 1994; 3: 1935-7.

10. Zheng F, Chevalier JA, Zhang LQ, et al. An Hphl polymorphism in the E-selectin gene is associated with premature coronary artery disease. Clin Genet 2001; 59: 58-64.

11. Marteau JB, Sass C, Pfister M, et al. The Leu554Phe polymorphism in the E-selectin gene is associated with blood pressure in overweight people. J Hypertens 2004; 22: 305-11.

12. Haidari M, Hajilooi M, Rafiei AR, et al. E-selectin genetic variation as a susceptibility factor for ischaemic stroke. Cerebrovasc Dis 2009; 28: 26-32.

13. Wang X, Zhang J, Du X, et al. Association of A561C and G98T polymorphisms in E-selectin gene with coronary artery disease: a meta-analysis. PLoS One 2013; 8: e79301.

14. Wu Z, Lou Y, Lu L, et al. Heterogeneous effect of two selectin gene polymorphisms on coronary artery disease risk: a meta-analysis. PLoS One 2014; 9: e88152.

15. Wenzel K, Stahn R, Speer A, et al. Functional characterization of atherosclerosis-associated Ser128Arg and Leu554Phe E-selectin mutations. Biol Chem 1999; 380: 661-7.

16. Watson SR, Imai Y, Fennie C, et al. The complement binding-like domains of the murine homing receptor facilitate lectin activity. J Cell Biol 1991; 115: 235-43.

17. Shan $\mathrm{H}$, Zhang $M$, Zhang M, et al. Association of rs 5368 and rs3917406 polymorphisms in E-selectin gene with premature coronary artery disease in Chinese Han population. Int J Clin Exp Med 2015; 8: 4387-92.

18. Nasibullin TR, Timasheva YR, Sadikova RI, et al. Genotype/allelic combinations as potential predictors of myocardial infarction. Mol Biol Rep 2016; 43: 11-6.

19. Gorący J, Gorący I, Kaczmarczyk M, et al. Low frequency haplotypes of E-selectin polymorphisms G2692A and C1901T give increased protection from coronary artery disease. Med Sci Monit 2011; 17: CR334-40. 
20. Miller MA, Kerry SM, Dong Y, et al. Circulating soluble E-selectin levels and the Ser128Arg polymorphism in individuals from different ethnics groups. Nutr Metab Cardiovasc Dis 2005; 15: 65-70.

21. Paterson AD, Lopes-Virella MF, Waggott D, et al. Genome-wide association identifies the $A B O$ blood group as a major locus associated with serum levels of soluble E-selectin. Arterioscler Thromb Vasc Biol 2009; 29: 1958-67.

22. Roldan V, Marin F, Lip GY, Blann AD. Soluble E-selectin in cardiovascular disease and its risk factors. A revive of the literature. Thromb Haemost 2003; 90: 1007-20.

23. Lawrence MB, Bainton DF, Springer TA. Neutrophil tethering to and rolling on E-selectin are separable by requirement for L-selectin. Immunity 1994; 1: 137-45.

24. Galkina E, Ley K. Vascular adhesion molecules in atherosclerosis. Arterioscler Thromb Vasc Biol 2007; 27: 2292-301.

25. Ley K, Allietta M, Bullard DC, Morgan S. Importance of E-selectin for firm leukocyte adhesion in vivo. Circ Res 1998; 83: 287-94.

26. Liao B, Chen K, Xiong W, et al. Relationship of SELE A561C and G98T variants with the susceptibility to CAD. Medicine 2016; 95: e1255.

27. Schaid DJ. Evaluating associations of haplotypes with traits. Genet Epidemiol 2004; 27: 348-64.

28. Hwang SJ, Ballantyne CM, Sharrett AR, et al. Circulating adhesion molecules VCAM-1, ICAM- 1 , and E-selectin in carotid atherosclerosis and incident coronary heart disease cases: the Atherosclerosis Risk In Communities (ARIC) study. Circulation 1997; 96: 4219-25.

29. Squadrito F, Saitta A, Altavilla D, et al. Thrombolytic therapy with urokinase reduces increased circulating endothelial adhesion molecules in acute myocardial infarction. Inflamm Res 1996; 45: 14-19.

30. Atalar E, Aytemir K, Haznedaroğlu I, et al. Increased plasma levels of soluble selectins in patients with unstable angina. Int J Cardiol 2001; 78: 69-73.

31. Suefuji $H$, Ogawa $H$, Yasue $H$, et al. Increased plasma level of soluble E-selectin in acute myocardial infarction. Am Heart J 2000; 140: 243-8.

32. Jankowski P, Czarnecka D, Badacz L, et al. Practice setting and secondary prevention of coronary artery disease. Arch Med Sci 2018; 14: 979-87.

33. Wu S, Hsu LA, Teng MS, et al. Association of SELE genotypes/haplotypes with sE-selectin levels in Taiwanese individuals: interactive effect of MMP9 level. BMC Med Genet 2012; 13: 115.

34. Opdenakker G, Van den Steen PE, Dubois B, et al. Gelatinase $B$ functions as regulator and effector in leukocyte biology. J Leukoc Biol 2001; 69: 851-6.

35. Menotti A, Lanti A, Puddu PE, Krohout D. Coronary heart disease incidence in northern and southern European populations: a reanalysis of the seven countries study for a European coronary risk chart. Heart 2000; 84: 238-44.

36. Nelis M, Esko T, Mägi R, et al. Genetic structure of Europeans: a view from the North-East. PLoS One 2009; 4: e5472. 